\title{
Determination of the magnetic separator separation function
}

\author{
Evgeniy Kharlamov*
}

Moscow State University of Civil Engineering, Yaroslavskoe shosse, 26, Moscow, 129337, Russia

\begin{abstract}
The article considers the design of magnetic separators for separation of iron-containing powders. The technology of separation of waste (tailings) of production by means of a separator of a boiling layer is offered. A technique for determining the separation coefficient and the dependence of the separation coefficient at different operating modes is presented. The efficiency of separation of the proposed separator above the traditional ones is shown.
\end{abstract}

\section{Introduction}

Currently, mining enterprises are mainly used wet scheme of separating raw materials and drum separators $[1,2]$. For wet magnetic separation of strongly magnetic ores are used in drum-type separators PBM (Fig. 1) with a multipolar system of permanent magnets and is available in three versions: with direct-flow, counter flow and semi-counter flow baths [3, 4]. Through the extractional zone from the direct-flow separators passes the entire volume of load, and semi-counter flow and counter flow- non-magnetic part. Direct-flow separators are used for materials with the grain size of $6 \mathrm{~mm}$ or less, counter flow ones - material fineness $2 \ldots 3 \mathrm{~mm}$ and less, semi-counter flow ones - for material with particle size of 0.3 $\mathrm{mm}$ or less $[5,6]$.
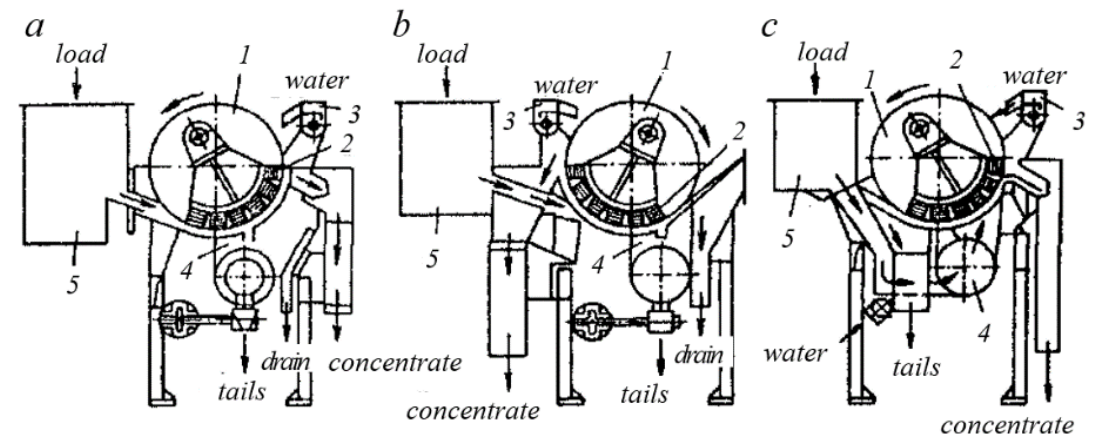

Fig. 1. Drum separator PBM-90/250 for wet enrichment of ores: $a$ - direct-flow bath; $b$ - counterflow bath; c-semi-counter bath: 1 -drum; 2 - six-pole magnetic system; 3 - overflow box for flush water, 4 - bath; 5 - loading box.

\footnotetext{
* Corresponding author: jkharlamov@mail.ru
} 
The separator 26-DS (Fig. 2) is a counter flow magnetic drum separator and is designed to enrich discharges of ball mills and classifiers [7]. The principle of operation of the separator 26-DS is: load pipe enters the feed box of the separator where two load nozzles are directed to the feeding tray and under the rotating drum. Magnetic particles in the magnetic field are attracted to the drum and moved to the edge of the magnetic system. There they are separated from the drum and discharged. Non-magnetic particles together with the water are discharged through the tail pipe.

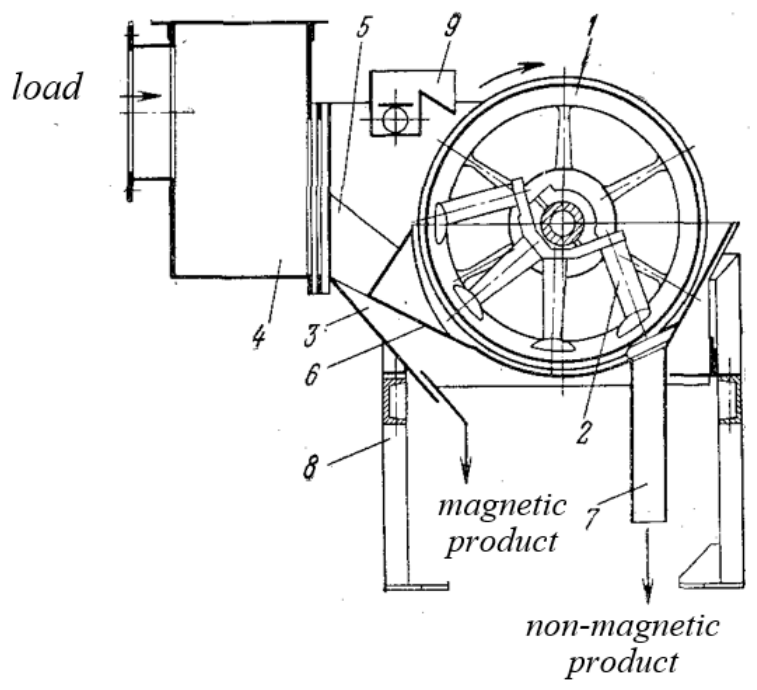

Fig. 2. Counter flow magnetic drum separator 26-DS: 1 - drum; 2 - magnetic system; 3 - bath; 4 loading box; 5 - feeding pipes; 6 - feeding tray; 7 - tail pipe; 8 - frame; 9 - sprinkles.

The above magnetic separators are separators for wet separation. They have the following negative drawbacks: the presence of water environment with a higher resistance compared to air; water environment has a strong opposition to the movement of magnetic and non-magnetic particles. As result the magnetic particles mix with nonmagnetic ones. When separating weakly magnetic materials is not observed magnetic flocculation (the formation of fortified units by the mutual attraction of the magnetized particles). It causes the need for feeding environment - water (waste water).

The source of waste water and recycled water are the drains dehydrating, washing machines and tailings. Polluting impurities are solids, hardness salts, heavy metal ions and organic substances. Untreated waste water that contains suspended impurities and aggregates, are the cause of the violation of the ecological system with all of its negative effects: shoal and dryen rivers, the vegetation dries, fades surrounding life [8,9]. Turnover out a huge area, where merge slimes from the enrichment process of iron ore $[10,11]$. For the treatment of waste water and impurities has applied mechanical, chemical, physicochemical and biochemical methods. All this leads to additional costs and cost of finished products.

An alternative of wet method is a dry method of separation. Environmental point of view dry magnetic separation is the most acceptable method of separation. The lack of feeding environment provides significant water savings, there is no need of sewage treatment and eliminates the need for sludges, which occupy a considerable area and negatively affect to the atmosphere and lithosphere $[12,13]$. Dry separation is the least energy intensive, as it is based on the natural property of magnetic attraction of iron ores. 


\section{Methods}

To improve the efficiency of dry separation tailings of metallurgical production is possible due to the overlapping processes of magnetic extraction of ferrous particles and air stiring separating the material $[9,14]$.

Based on these requirements we have developed and manufactured an experimental installation for studying the process of separation of a two component mixture. Photo of the current installation is shown in Fig. 3.

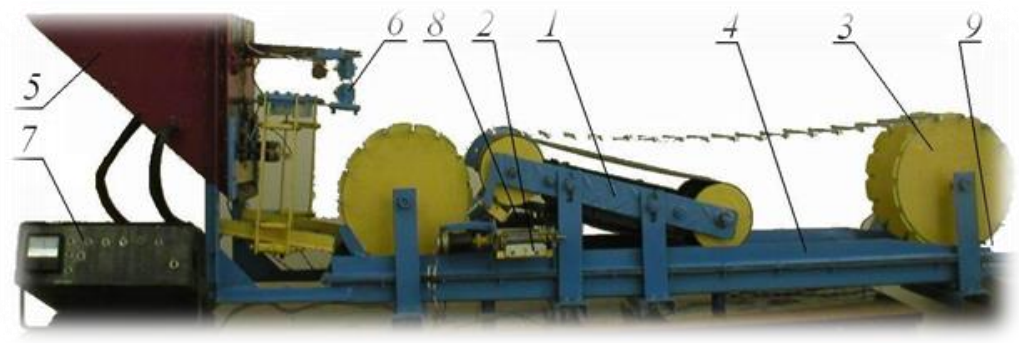

Fig. 3. Experimental installation: 1 - the belt conveyor; 2 - conveyor unloading magnetic particles; 3 - the scraper conveyor; 4 - the transporting tray; 5 - loading device; 6 - the stabilization system; 7 the control panel; 8 - the discharge zone of magnetic particles; 9 - the zone of unloading nonmagnetic particles.

The experimental installation consists of the following main components: the belt conveyor 1 , the conveyor unloading the magnetic particles 2 , the scraper conveyor 3 , the transporting tray 4 , loading device 5 , the stabilization system 6 , the control panel 7 , the discharge zone of the magnetic particles 8 and the zone of unloading non-magnetic particles 9. The belt conveyor is designed to extract from the mixture of the magnetic particles. Traction circuit inside the belt conveyor along the lower branches of the conveying tape hinged block of magnets. Belt conveyor set at an angle relative to the transporting tray. This situation creates a belt conveyor in relation to moving in the transported tray of a mixture of variable magnetic field from the minimum at the beginning of the extraction to the maximum at an end of the drum. Accordingly, the extraction process of the particles occurs during the period of movement of the mixture under the active section of the belt conveyor in the beginning to extract strongly magnetic particles and decreasing the magnetization of the particles.

Scraper conveyor is designed to stabilize the motion of the mixture on the transporting tray to discharge non-magnetic particles and does not prevent the process of separation of a mixture of magnetic particles. Scraper conveyor contains made of non-magnetic materials two-chain traction body with the attached scrapers. The transporting tray bottom made in the form of a box. The upper surface of the transporting tray made of permeable to air of the porous material. Passing through the porous material air forms a layer of compressed air between the surface of the porous material and the mixture, with the result that the air passes through the mixture creates the effect of fluidization. Separation of a mixture into fractions in the regime of fluidization increase the mobility of the particles. This increases the probability of their capture by the magnetic field, improves the recovery ratio of magnetic particles that reduces the possibility of occurrence of particles on the partition and coefficient their resistance movement of the particles.

The separator fluidized bed dry separation works in the following way. Through the pipes into the hollow elements of the transporting tray is served working agent - the air that passes through the porous material and creates in the contact zone of a material with a porous layer of compressed air, which reduces the coefficient of friction in 3...6 times and provides a steady movement of material in the direction of unloading. The initial mixture of 
magnetic and non-magnetic particles is fed into the hopper of the loading device. The mixture enters through the open gate grooves in the front wall of the hopper of the loading device on the vibrating tray. The vibrating tray makes reciprocating motion directed perpendicular to the motion of the mixture. The vibrating tray is distributed the raw material mixture across the width of the load. Distributed the raw material mixture directed to the conveying tray of the separator of fluidized bed and move by scrapers of the scraper conveyor to the zone of non-magnetic particles. The lower branch of the belt conveyor moves in counter flow mix and draws from a mixture of magnetic particles. The process of extraction of the particles proceeds as follows. In early extract highly-magnetic particles, and then by decreasing magnetization of the particle. The extracted particles confined by the magnetic field are moved with the belt towards the area of unloading magnetic particles, which transport them outside the unit. Non-magnetic particles are transported further to a zone of unloading non-magnetic particles.

\section{Results}

Extracting the ability of the separator of the fluidized bed depends on the characteristics of the magnetic system, physical and mechanical properties of the particles of the separated mixture and the geometric parameters of the working area $[15,16]$. Recovery ratio of magnetic particles $\eta$ is determined by the ratio of their volumes at the entrance to the active zone $G(x A)$ and on the way out of it $(x B)$ (Fig.4):

$$
\eta=1-\frac{G\left(x_{B}\right)}{G\left(x_{A}\right)} .
$$

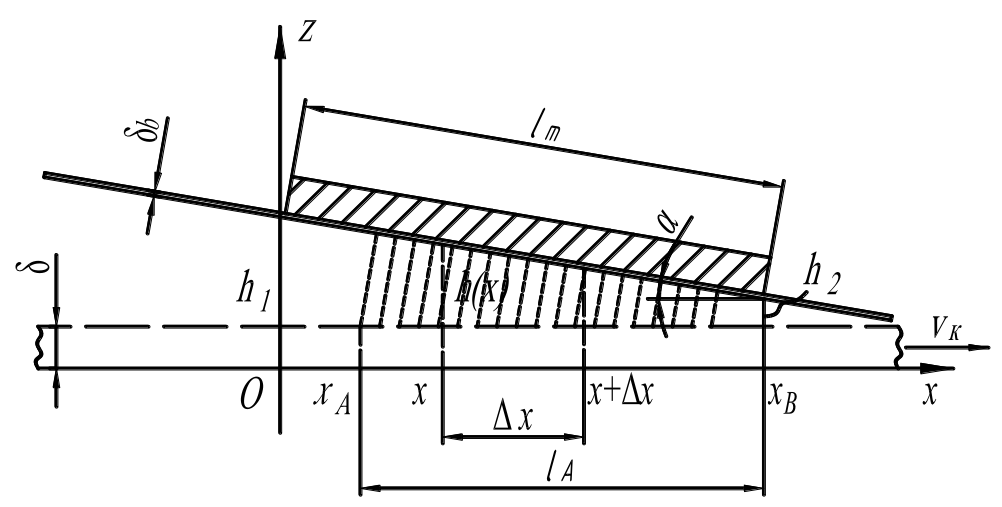

Fig. 4. Calculation scheme for determining of extraction ratio of magnetite particles.

Extracting the particles in the separation zone causes aero disperse stream of magnetic particles. They is moved along the working area of the separator fluidized bed at a speed $v$ of the scraper conveyor. The concentration of magnetic particles in this stream are distributed across the height of the working area is very uneven: it sharply decreases from a maximum value in a boiling layer to a minimum value in the zone of deposition to the transport conveyer belt.

Consider a part of the working area of the separator fluidized bed between the cross sections passing through the points $x$ and $x+\Delta x$ (see Fig. 4). As a result of precipitation of magnetic particles, their concentration decreases from $C(x)$ to $C(x+\Delta x)$. Material balance equation of magnetic particles for the selected part of the working area: 


$$
G(x)-G(x+\Delta x)=\chi C(x) v_{1} b \frac{\Delta x}{\cos \alpha}=\chi \frac{G(x) v_{1} \Delta x}{h(x) v \cos \alpha} .
$$

Here, $\Delta x / \cos \alpha$ - a part of the transporting conveyor belt, corresponding $\Delta x, G(x)-$ volume of magnetic particles passing through the cross section of the working area corresponding to a coordinate $x$ :

$$
G(x)=C(x) b h(x) v,
$$

where $h(x)$ - the height of this section:

$$
h(x)=h_{1}-x \operatorname{tg} \alpha,
$$

where $v_{1}$ - the precipitation velocity of particles on the transport conveyer belt; $b$ - the width of the cross section; $\alpha$ - the angle of scraper conveyor; $v$ - the separated material speed.

Expressing the change volume magnetic particles through its differential, we obtain:

$$
G(x)-G(x+\Delta x)=-(G(x+\Delta x)-G(x)) \approx-d G(x),
$$

and dividing the variables in equation (1), we get:

$$
\frac{d G}{G}=-\frac{\chi v_{1} d x}{v\left(h_{1}-x \operatorname{tg} \alpha\right) \cos \alpha} .
$$

Integrated equation (6), we obtain:

$$
\left.\ln G\right|_{G\left(x_{A}\right)} ^{G\left(x_{B}\right)}=\left.\frac{\chi v_{1}}{v \sin \alpha} \ln \left(h_{1}-x \operatorname{tg} \alpha\right)\right|_{x_{A}} ^{x_{B}} .
$$

From relation (7) follows:

$$
\frac{G\left(x_{B}\right)}{G\left(x_{A}\right)}=\left(\frac{h_{1}-x_{B} \operatorname{tg} \alpha}{h_{1}-x_{A} \operatorname{tg} \alpha}\right)^{\frac{x v_{1}}{v \sin \alpha}} .
$$

Substituting expression (8) in the formula (1), we obtain a relation for the fractional extraction ratio of magnetic particles:

$$
\eta(d)=1-\left(\frac{h_{2}}{h_{2}+l_{A} \operatorname{tg} \alpha}\right)^{\frac{x v_{1}}{v \sin \alpha}} .
$$

For incoming in the formula (6) length of the active portion of the working area $l_{A}$ we obtain:

$$
l_{A}=-\frac{h_{2}-1,115 \delta}{\operatorname{tg} \alpha}-\frac{1}{2 c \sin \alpha} l_{n}\left(\frac{\rho g \cos \alpha}{\mu_{0} \chi c H_{0}^{2}}\right) \text {. }
$$

For the experimental fluidized bed separator $\left(h_{2}=0,015 \mathrm{~m}, \delta=0,003 \ldots 0,013 \mathrm{~m}, v=\right.$ $0,012 \ldots 0,028 \mathrm{mps}, \alpha=10 \ldots 20^{\circ}, c=26,17 \mathrm{~m}^{-1}, H_{0}=37 \mathrm{kApm}, \chi=6,25, \rho=5260 \mathrm{kgpm}^{3}$, $\Phi=1,65, g=9,81 \mathrm{mps}^{2}, \mu_{0}=4 \pi \cdot 10^{-7}(\mathrm{~kg} \cdot \mathrm{m}) /\left(\mathrm{s}^{2} \cdot \mathrm{A}^{2}\right), d_{i}=\{2,7 ; 8,3 ; 17,2 ; 31,7 ; 55\}$ micron, $\mu=$ 
$\left.1,8 \cdot 10^{-5} \mathrm{~Pa} \cdot \mathrm{s}, \delta_{b}=0,003 \mathrm{~m}\right)$ parameter $l_{A}$ and the precipitation velocity of particles on the transport conveyer belt $v_{1}$ (formula 10)) takes the form:

$$
\begin{gathered}
l_{A}=-\frac{0,015-1,115 \delta}{\operatorname{tg} \alpha}-\frac{\ln (0,183 \cos \alpha)}{52,34 \sin \alpha}, \\
v_{1}=4,472 \cdot 10^{-4} d^{2},
\end{gathered}
$$

where the particle size $d$ should be set in microns.

Taking into account formulas (11) and (12) the expression (9) can be rewritten in the form

$$
\eta(d)=1-\left(\frac{0,015}{1,115 \delta-\frac{\ln (0,183 \cos \alpha)}{52,34 \cos \alpha}}\right)^{\frac{4,472 \cdot 10^{-4} d^{2} \chi}{v \sin \alpha}} .
$$

Full recovery of magnetite particles $\eta$ is determined by the fractional factors $\eta\left(\bar{d}_{i}\right)$ and disperse composition of the particles:

$$
\eta=\sum_{i} \eta\left(\bar{d}_{i}\right) f_{i}
$$

Comparison of theoretical and experimental values of the full coefficient of extraction of magnetic particles shows that the magnitude of the coefficient of heterogeneity of the distribution of their concentration in the working area of the separator fluidized bed $\chi$ depends mainly on the thickness of the partial mixture in the initial bound state $\delta$. As a result of processing of experimental data obtained the following dependence:

$$
\chi(\sigma)=50 \delta^{2}-0,15 \delta+0,0224 .
$$

Substituting the formula (15) in (13) we obtain the final expression for the fractional extraction ratio of magnetic particles in an experimental fluidized bed separator:

$$
\eta(d)=1-\left(\frac{0,015}{1,115 \delta-\frac{\ln (0,183 \cos \alpha)}{52,34 \cos \alpha}}\right)^{\frac{4,472 \cdot 10^{-4} d^{2}\left(50 \delta^{2}-0,15 \delta+0,0224\right)}{v \sin \alpha}} .
$$

From formulas (6) and (7) we can see that the recovery ratio of magnetic particles depends on their physic-mechanical properties (density, size, shape, magnetic permeability), the characteristics of the magnetic system (its size, magnetic field strength, spacing of poles), the constructive-technological parameters of fluidized bed separator (dimensions, tilt angle of the magnetic system, the thickness of the partial layer of the mixture, the speed of movement of the scraper conveyor), but also properties of the air environment (temperature, viscosity).

To confirm the above presented experimental installation we conducted an experimental study. The used material is tails Lebedinsky mining plant. In the course of the experiment changed the following parameters: layer thickness $\delta$ of the separating material; moving velocity $v$ of the separating material; the angle of inclination of a belt conveyor $\alpha$ and the magnetic field strength $H$. 


\section{Conclusions}

In Fig. 5 shows the dependence of the influence of the layer thickness $\delta$ of the separating material on the separation efficiency $\eta$ with different values of the moving velocity of the separating material $v$, the constant angle of inclination of a belt conveyor $\alpha=15^{\circ}$ and magnetic field $H=40 \mathrm{kApm}$ Here we can see that all the curves have increasing character, i.e. with increasing layer thickness of the separating material parameter $\delta$ increases the separation efficiency.

Analyzing graphs (figure 5) we can conclude that the maximum value of the separation efficiency $\eta$ in the experiment is achieved when the velocity of the iron-containing composition $v=0.02 \mathrm{mps}$ and the layer thickness of $\delta$ equal to $14 \mathrm{~mm}$ and $\eta$ is of $91.23 \%$.

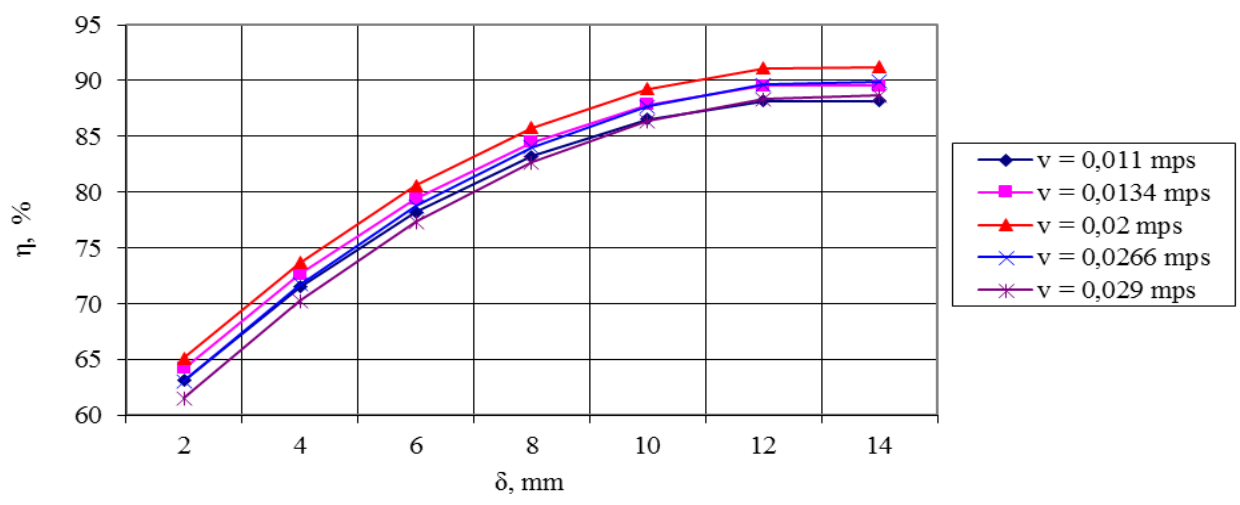

Fig. 5. Dependence of the separation efficiency $\eta$ of the layer thickness of the separating material $\delta$ under different values of the moving velocity $v$ of the separating material, constant angle of inclination of a belt conveyor $\alpha=15^{\circ}$ and magnetic field $\mathrm{H}=40 \mathrm{kApm}$.

Fig. 6 shows the dependence of the effect of velocity of movement $v$ of the separating material on the separation efficiency $\eta$ under different values of the angle $\alpha$ of inclination of a belt conveyor, a constant layer thickness of the separating material $\delta=8 \mathrm{~mm}$ and magnetic field $\mathrm{H}=40 \mathrm{kApm}$ Here we can see that all the dependences are of an extreme nature in the studied range of variation of the factors.

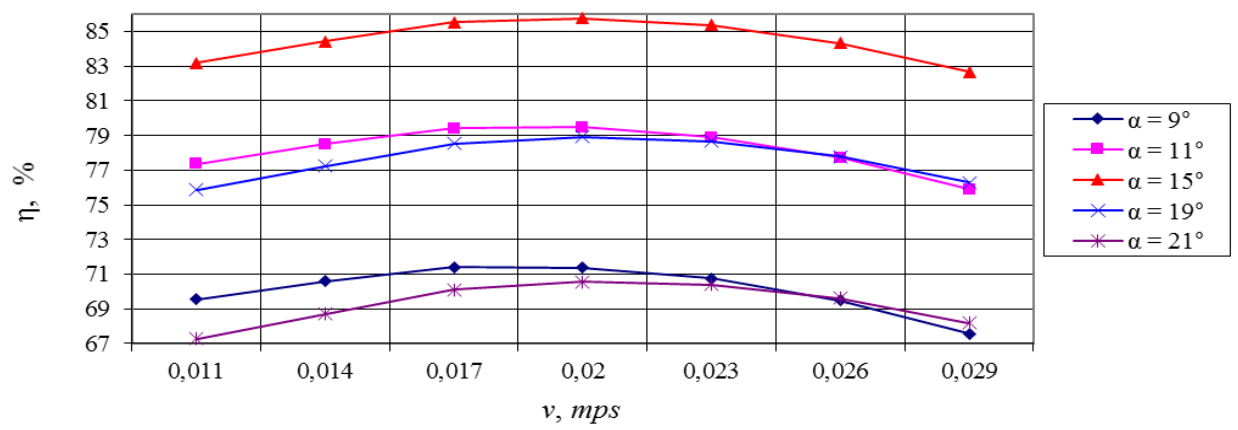

Fig. 6. Dependence of the separation efficiency $\eta$ from of the moving velocity of the separating material $v$ under different values of the angle of inclination of a belt conveyor $\alpha$, a constant layer thickness $\delta=8 \mathrm{~mm}$ and magnetic field $H=40 \mathrm{kApm}$.

Analyzing graphs we can conclude that the maximum value of the separation efficiency $\eta$ is achieved when the velocity of the separating material $\mathrm{v}=0.02 \mathrm{mps}$, angle of installation of a belt conveyor $\alpha=15^{\circ}$ and $\eta$ is $85.77 \%$. 
With the aim of improving environmentally friendly production of materials metallurgical production under separation tailings with a high degree of effectiveness proposed scheme dry processing. It is proved that for these purposes can be applied by the magnetic principle of extraction of particles and airs tirring of separating material.

For conducting experiment we designed and constructed experimental installation of fluidized bed separator.

Experimental studies confirm that the proposed in article separator fluidized bed dry separation is more effective in comparison with traditional separators. Efficiency exceeds $90 \%$.

\section{References}

1. R.P. Bhagat, P.N. Pathak, IJMP. 47. (1996)

2. T. Kaljuvee, R. Kuusik, M. Veiderma, IJMP. 43. (1995)

3. M. Lovás, I. Murová, A. Mockovciaková, N. Rowson, S. Jakabský, SPT. 31. (2003)

4. H. Zhang, L. Chen, J. Zeng, L. Ding, J. Liu, SST. 50. (2015)

5. V.A. Simakov, V.E. Isaev. IM. 51. (2015)

6. A.M. Turkenich, E.S. Lapshin, V.I., MES. 8. (1997)

7. V.E. Potapenko, D.I. Suvorova, V.V. Tyuryukhanova, RIC. 22. (1981)

8. V.F. Skorokhodov, S.P. Mesyats, S.P. Ostapenko, EM. 1. (2011)

9. E.V. Kharlamov, R.R. Sharapov, V.V. Yadykina, IJAER. 10. 24. (2015)

10.I.V. Melik-Gaykazov, Yu.G. Druz, V.A. Shchetkin, Yu.A. Komyagina, V.F. Skorokhodov, EM. 2. (2012)

11. E. Korol, N. Shushunova, WMCAUS 2016. (2016)

12. J. Oshitani, G.V. Franks, M. Griffin, APT. 21. (2010)

13. K.E. Anatolevna, K.P. Borisovich, IJAER. 10. (2015)

14. R. Sharapov, P. Kapyrin, S. Lozovaya, V. Yadykina, A. Agarkov, IPICSE 2016. (2016)

15. N.S. Sevryugina, Theoretical Foundation of Civil Engineering MATEC Web of Conferences 117, 00151 (2017)

16. N.S. Sevryugina, E.A. Volkov, E.P. Litovchenko, MAS. 8. (2014) 